\section{An Augmented Reality Learning Environment for Informal Geoinformatics Education}

GI_Forum 2021, Issue 2

Page: $3-17$

Full Paper

Corresponding Author: josef.buchner@uni-due.de DOI: 10.1553/giscience2021_02_s3

Josef Buchner1, Peter Michael Jeremias², Nikola Kobzare², Lelia König², Sebastian Oberreiter ${ }^{2}$, Stefan Reiter ${ }^{2}$ and Bernd Resch²

IUniversity of Duisburg-Essen, Germany

2University of Salzburg, Austria

\begin{abstract}
In this paper, we describe an interactive learning environment for geoinformatics enriched by Augmented Reality (AR) that was designed by researchers and practitioners using a design-based research approach. In total, four AR modules were developed to engage learners physically, cognitively and emotionally in the learning process. AR is thus used not merely as a visualization technology, but also to initiate and scaffold learning activities. To address the known challenges of learning with AR, we designed our learning environment to be used by teams of students. This can counteract the problem of cognitive overload, or frustration due to technical problems. We examined our technological and pedagogical framework in an evaluation with 12 learners. The results show that our design did not lead to cognitive overload; technological aspects, such as usability, were also evaluated positively. However, we also detected possible improvements to our design, such as using AR glasses to free the hands for task completion. Overall, it was shown that AR is a good way to expand traditional learning opportunities with digital ones and to enable holistic learning experiences.
\end{abstract}

\title{
Keywords:
}

geoinformatics, augemented reality, collaborative learning, education, interactive learning

\section{Introduction}

In science education, students often struggle to understand the complexity and interrelationships of natural phenomena, since these are not immediately perceivable or tangible. Consequently, educators increasingly report misconceptions on the part of students, and a decline in their interest and motivation with regard to science learning (see e.g. Sahin \& Yilmaz, 2020). To counteract this, researchers are calling for science learning to be studentcentred and to use an active learning paradigm (Deslauriers et al., 2011), in which learners become (co-)designers of the learning process by asking questions, hypothesizing, and planning experiments with which to test predictions (Goff et al., 2020). The use of technology can support these self-regulated learning activities in a variety of ways, including just-in-time 
feedback, scaffolding, making non-visible phenomena visible, and giving students the feeling of experiencing an authentic situation that requires real-life problem-solving (Yannier et al., 2020).

In recent years, augmented reality (AR) technology has proved particularly promising for this purpose because, in contrast to immersive virtual reality (iVR), reality is not completely replaced but enhanced by digital elements (Cheng \& Tsai, 2013). Hence, learners can still explore the real world, but at the same time they can see underlying relationships; helpful information can be displayed directly on an object, and students can even make changes to both the object and the information that are displayed in real time and can thus be observed (Dunleavy et al., 2009). Moreover, many studies have demonstrated that learners find AR motivating, satisfying and interesting, which in turn has led to improved attitudes towards science learning (Ibáñez \& Delgado-Kloos, 2018).

In addition to these potentials, researchers also point to challenges when using AR in learning environments. From a technical perspective, particular attention must be paid to the usability of the AR application employed. If the operation is perceived as difficult and complicated, negative effects on both affective and cognitive learning outcomes can be observed. For example, the usefulness of the technology for one's own learning is then questioned, satisfaction decreases, and further use of the application becomes less likely (Ibili et al., 2019). Another factor that must be considered is cognitive load. AR learning environments integrate different media and require self-directed learning, which could lead to cognitive overload for students (Lai et al., 2019).

Based on these affordances of science education and AR learning, we developed an AR learning environment for geoinformatics using a design-based research approach. In this study, we present a design consisting of four AR modules. In addition, we report the results of our initial evaluation, in which 12 learners tested the first iteration of the modules. The goal of the evaluation was to find out whether the AR modules are perceived as cognitively demanding, and whether the technical framework used is suitable, acceptable to students, and perceived as user-friendly.

\section{Design Process}

The development and design of the AR-enhanced learning modules for geoinformatics was part of the ARED project (Augmented Reality for Education), which is being carried out in cooperation with the iDEAS:lab Salzburg1. In this project researchers from the fields of educational technology and geoinformatics, practitioners, university students and AR developers collaborate to design meaningful and exciting educational experiences for school students and the public. This merging of research and practice can help to ensure that innovative educational practice persists beyond the duration of the project itself (Bell, 2004). One approach that is explicitly suitable for projects with theory-practice linkages is the designbased research approach (DBR), in which concrete educational problems are first analysed together with practitioners, and concrete solutions for the problems are then worked on

\footnotetext{
${ }^{1}$ https://ideaslab.sbg.ac.at
} 
(McKenney \& Reeves, 2019). Next, the solution is tested and evaluated in practice. Since the DBR procedure is cyclical, the findings from the evaluation are used to make any necessary adjustments. At the end of this process, design principles are derived that expand both the theoretical and the practical understanding of teaching and learning (McKenney \& Reeves, 2019).

\subsection{Development of the pedagogical framework}

Following the generic model of DBR (McKenney \& Reeves, 2019), first we analysed and explored the learning activities and materials that are already to be found in the iDEAS:lab Salzburg. This phase consists of a visit to the iDEAS:lab and intensive discussions with practitioners, including educators and ICT experts. This helps the research team to understand the educational problem(s). For example, one installation in the iDEAS:lab aimed to visualize the functional principle of satellite-based positioning, a 3-dimensional phenomenon. However, at the beginning of the project this phenomenon was introduced using 2-dimensional representations on a computer screen, making it hard for learners to understand it. As a team, we identified three other installations with similar deficiencies, such as a lack of illustration of spatial concepts, and either insufficient information or a presentation of information that was too demanding, cognitively, to perform learning tasks effectively. These inadequacies can come to light thanks to experience and evidence from the science of instruction and can be mitigated by redesign. According to cognitive load theory (CLT) (Sweller et al., 2019), well-designed instructional materials can help learners to manage the cognitive demand induced by a learning activity and thus promote better learning. For example, the split-attention effect states that information to be learned or that is needed to perform a task should be presented close in space and time to the equipment and materials required (Ayres \& Sweller, 2014). Furthermore, effective learning environments are ones that provide tasks which engage learners cognitively, emotionally and physically (Domagk et al., 2010). For example, Lindgren et al. (2016) developed a mixed-reality platform for learning about the path of asteroids and its consequences for planets. Learners simulated the path of the asteroid through movements with their bodies and immediately observed how their movements affected the asteroid's speed and trajectory. Compared to a control group, the learners in the mixed-reality simulation (i.e. which included movements) performed significantly better on a post-test. Lindgren et al. (2016) conclude that body movements are a promising way for supporting learners' understanding of spatial phenomena.

Based on the practical and theoretical considerations explored, we started the second phase of the DBR model, namely design and construction, and generated solutions to address the deficiencies we have mentioned. We decided to use AR, as this technology had been used in previous studies to address similar problems. AR is defined as the computer-supported extension of the real world by virtual objects, resulting in a situation in which reality and virtuality coexist in both time and space (Azuma et al., 2001). Sommerauer and Müller (2014) used $\mathrm{AR}$ in a museum to visualize mathematical concepts in a more effective way, for example by integrating 3-dimensional objects or displaying additional information directly on a realworld object. In a similar way, Chang and Hwang (2018) used AR to provide immediate feedback to learners in a physics class. For example, the students conducted an experiment about magnetic fields using physical materials, and with the help of AR they could see whether 
their constructions were correct. In Yoon et al. (2017), visualizing the invisible in science education, such as air streams, was found to be a major potential of AR when compared to more traditional instructional materials and methods. However, researchers also reported negative effects of AR-enhanced learning environments that need to be considered in their design. Ibili et al. (2019) point out that any AR system implemented must be easy to use. Otherwise, frustration can arise, which has a negative effect on learning. Additionally, the selfdirected nature of AR learning environments, combined with technical affordances, can be cognitively demanding and consequently may result in poor learning (Dunleavy et al., 2009; Lai et al., 2019). To overcome these issues, we designed the AR modules in such a way that they can also be worked on collaboratively. Collaborative learning is not only highly effective but can also reduce the risk of cognitive overload (Janssen \& Kirschner, 2020). Learners help each other with the tasks, and technical problems are also solved as a team.

With these potentials and challenges of AR-enhanced learning environments in mind, we constructed four AR modules which include learning tasks and virtual elements that can address the shortcomings mentioned earlier, and therefore contribute to solving our educational problem(s). An overview of the AR modules is given in Table 1.

Table 1: Overview of the modules, problems identified, solutions developed and learning tasks

\begin{tabular}{|c|c|c|c|}
\hline Module & Problem & Solution & Learning task(s) \\
\hline $\begin{array}{l}\text { Visualizing the } \\
\text { functional } \\
\text { principle of } \\
\text { GNSS }\end{array}$ & $\begin{array}{l}\text { Limited } \\
\text { representation of a } \\
\text { 3D phenomenon. }\end{array}$ & $\begin{array}{l}\text { Visualization of the } \\
\text { signal processing and } \\
\text { trilateration using 3D } \\
\text { AR objects }\end{array}$ & $\begin{array}{l}\text { Collaborative } \\
\text { documentation of the } \\
\text { satellite signals and } \\
\text { their positions }\end{array}$ \\
\hline $\begin{array}{l}\text { Construction of } \\
\text { a sensing } \\
\text { station }\end{array}$ & $\begin{array}{l}\text { Cognitively } \\
\text { demanding } \\
\text { instructional } \\
\text { manual }\end{array}$ & $\begin{array}{l}\text { AR-based guidance } \\
\text { directly on the } \\
\text { hardware }\end{array}$ & $\begin{array}{l}\text { Construction of the } \\
\text { sensing station }\end{array}$ \\
\hline $\begin{array}{l}\text { Performing } \\
\text { geospatial tasks } \\
\text { with the AR } \\
\text { sandbox }\end{array}$ & $\begin{array}{l}\text { Lack of information } \\
\text { to set the heights } \\
\text { of the sand } \\
\text { landscapes in } \\
\text { relation to each } \\
\text { other }\end{array}$ & $\begin{array}{l}\text { Complementing the } \\
\text { sandbox with an AR- } \\
\text { based legend } \\
\text { containing elevation } \\
\text { scales }\end{array}$ & $\begin{array}{l}\text { Collaborative } \\
\text { landscape modelling, } \\
\text { and comparison with } \\
\text { locally relevant } \\
\text { information }\end{array}$ \\
\hline $\begin{array}{l}\text { Spatial-temporal } \\
\text { distribution of } \\
\text { geo-social media }\end{array}$ & $\begin{array}{l}\text { The non-visibility } \\
\text { of social media } \\
\text { postings and } \\
\text { location }\end{array}$ & $\begin{array}{l}\text { Visualization of } \\
\text { social media posts as } \\
\text { AR objects directly on } \\
\text { a physical map }\end{array}$ & $\begin{array}{l}\text { Collaborative search } \\
\text { for specific } \\
\text { locations, e.g. } \\
\text { streets with high } \\
\text { volume of traffic }\end{array}$ \\
\hline
\end{tabular}

\subsection{Technical development of the AR modules}

In this study, we chose to use the Wikitude software development kit (SDK), version 8.10, which provides a cross-platform AR environment and is combinable with multiple development engines. We used the Wikitude SDK primarily for the integration of virtual elements into the AR-based visualization, making use of Wikitude's image- and object-tracking capabilities, which we realized differently for each module. The common guiding principle in 
our design was to use markers of various kinds and thus to transform real-world objects into recognizable virtual objects.

To create a comprehensive AR experience for our learners, we designed and generated virtual objects to be displayed in the AR environment. Unity supports the development of VR applications and allows the use of the Wikitude SDK as an add-on package. For this purpose, we chose Unity version 2018.4.22f1 because of its extensive functionalities, which we required for the goals of our research, and due to its strong user-base and support. The Unity Editor was used to generate virtual objects, design actions (including triggers and loops), and to build the AR application. In practice, following the Module functional principles of GNSS (Section 3.1) as an example, we used this AR foundation as follows. In Unity, satellite vectors were imported from the library and positioned; spheres and signal features were created and integrated into an animation of the positioning workflow. Using the Wikitude SDK, a QR code was generated, which served as a marker for the AR app and was defined as the trigger for the application.

To give the reader a more precise illustration of the AR modules, we describe two of them in the following section. A detailed description of the other two modules can be found in the Appendix, and a video demonstration of all modules can be found here: $\mathrm{t} 1 \mathrm{p} . \mathrm{de} / \mathrm{aredu}$

\section{Sample AR modules}

\subsection{Visualizing the functional principle of GNSS}

Signal processing and the principle of trilateration are key to satellite-based positioning (Teunissen \& Montenbruck, 2017). Although the GNSS communication principle is onedirectional, learners often assume bi-directional communication between satellites and mobile devices. Thus, transparently communicating the GNSS trilateration principle is key to understanding the technology, but it is typically limited by how 3-dimensional phenomena are represented in a 2-dimensional learning environment (e.g., a computer screen). Intersecting spheres that represent omnidirectional satellite signal propagation are difficult to visualize in a 2-dimensional representation, resulting in the risk of misunderstandings and of generating incorrect information. Using an AR-based 3-dimensional representation of the trilateration may simplify the learning of the underlying principle through an improved spatial perception of this process. Students' spatial imagination is thus supported through AR, which in turn leads to an increased understanding of more advanced topics, such as possible problems with signal propagation or possible location-specific inaccuracies.

In practical terms, the GNSS AR module functioned as follows. Upon successful detection of the marker (in this instance a QR code on the ceiling) by pointing the camera of a mobile device (e.g. a tablet) at it, the AR app visualizes four virtual satellites, and the signal transmission of each satellite is displayed schematically, in a single direction. The visualization clearly shows the intersection points of the distance spheres, representing potential GNSS position candidates. The three-dimensionality of these signal transmissions is underpinned by the use of a distance sphere around each satellite (the centre), which gradually increase in size to represent the distance to the satellite. The surfaces of these semi-transparent spheres and 
the schematic signal paths, represented as coloured lines, intersect at a certain point, which represents an exemplary GNSS position. In this use case, the QR code on the lab's ceiling emulates the elevated position of the satellites; the subsequent trilateration animation was designed to be seen from below the marker. After the students had generated new virtual objects, or acquired existing ones, the main focus was on the illustration of the positioning workflow. This included the correct placement of satellites in a circle around the marker, the definition of an exemplary positioning-target, and the animation sequence of spheres and signals. This animation element is especially important to ensure students' proper understanding of the workflow in GNSS. Figure 1 shows an illustration of the virtual elements in the preparatory phase (left), and of the in-app visualization in a real environment (right). A video demonstration of the GNSS module is available here: t1p.de/gps6

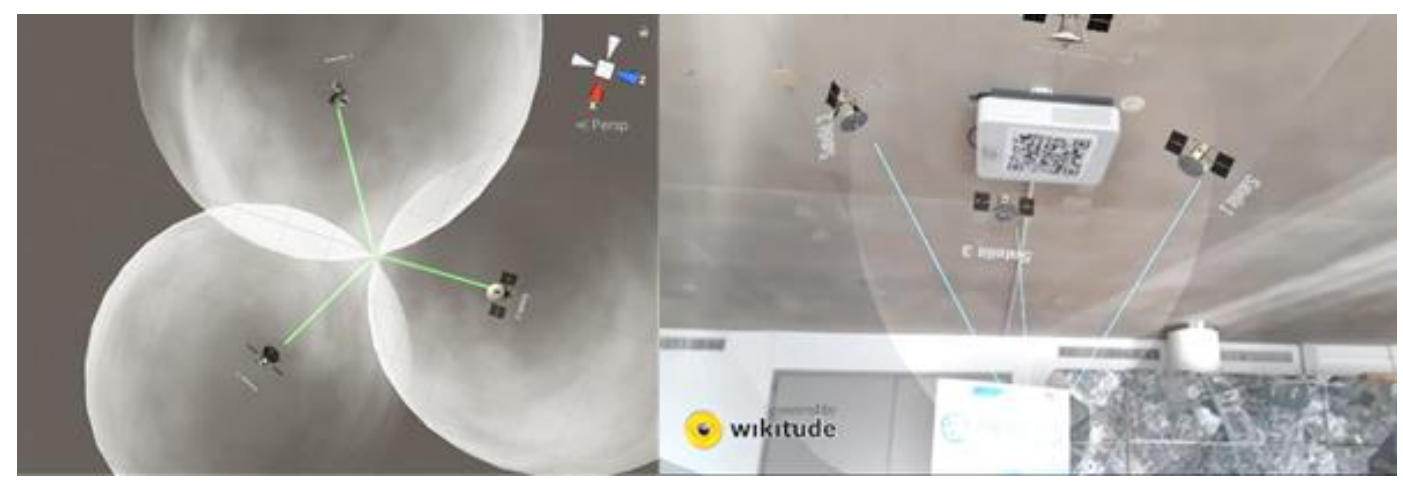

Figure 11: Illustration of the virtual elements (left) and of the in-app visualization in a real environment (right).

\subsection{Visualizing the spatial-temporal distribution of geo-social media}

Geo-social media have recently been widely used in scientific research in a variety of fields, from disaster management, epidemiology and urban planning, to communication science and political science. However, the geospatial nature and the wider implications of social media data and their usage (i.e. the benefits and risks of social media) are mostly not transparently communicated. In fact, geo-social media data can be used to extract thematically relevant information from personal data to investigate spatial-temporal phenomena, for example in urban science and urban planning (Resch et al., 2016). Using digital media in a citizen-centred approach to planning promotes the discussion of publicly relevant issues in a more inclusive development environment and the integration of the voices of members of the public.

The AR visualization created for our study aims to illustrate the spatial-temporal patterns of social media usage graphically together with the semantic content of social media posts, i.e. what opinions and sentiments people express at and about different locations. The cartographic challenge in this case was to find a balance between maintaining the overview of larger geographical areas for reasons of orientation in geographical space, and the detailed visualization of individual locations and social media posts, while also keeping information density at a digestible level. 
We therefore created an AR visualization that used a marker-based approach and existing physical maps as triggers. The maps also provided the spatial reference for the AR-based illustration of social media posts. Figure 2 shows these physical map elements (left), which are parts of the city of Salzburg. For reasons of information readability and spatial assignability, a simplified representation of the city was used (i.e., a graphically appealing city map). Sample posts were created as virtual objects, and position icons were placed on the digital representation of the map tile, used as the marker. As before, animation effects were used to represent positions of posts, which in this case was carried out by having parts of the texts rise vertically from the position icons. In this scenario, the marker was used not only as a trigger, but also as a base layer for the overlaid information - which is precisely what constitutes the novelty in the merged visualization of real and virtual objects (see Figure 2, right). A video demonstration of the geo-social media module is available here: $\mathrm{t} 1 \mathrm{p} . \mathrm{de} /$ mediar

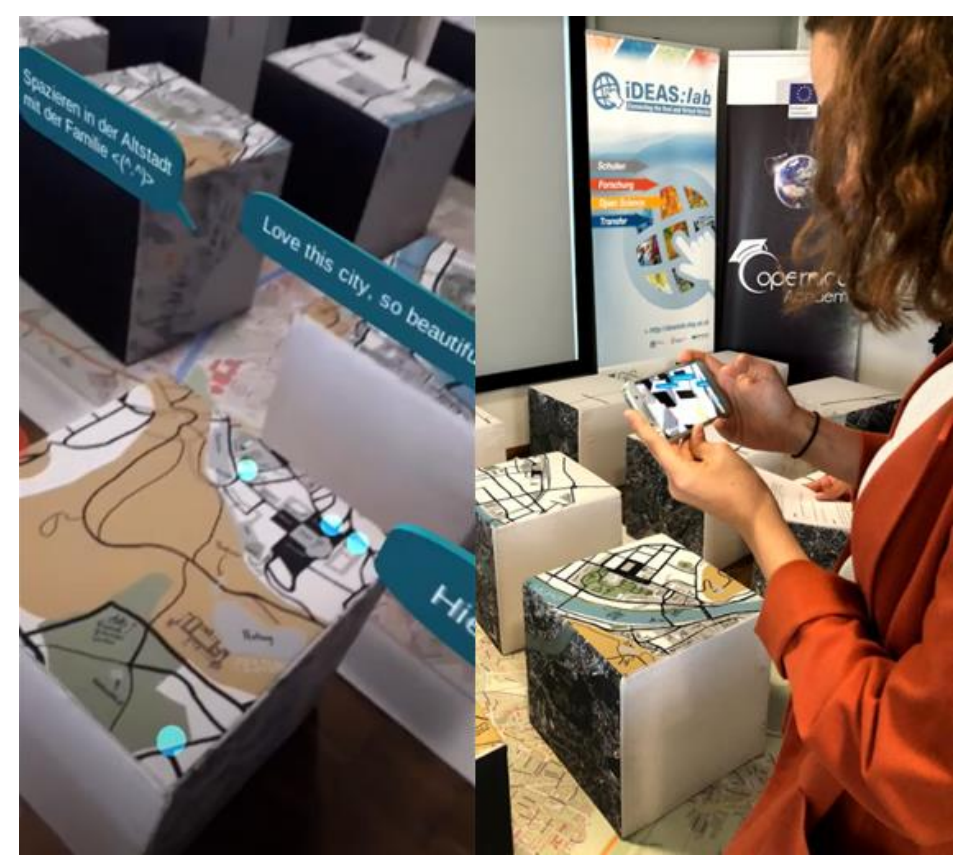

Figure 2: Physical map elements serve as markers (left), which are overlaid by virtual objects and displayed on the users' devices (right).

\section{Evaluation}

\subsection{Participants and instruments}

To test our first iteration, we recruited five women and seven men $(\mathrm{n}=12)$ with a mean age of $20.58(\mathrm{SD}=0.99)$. They participated as volunteers; they received no gratuity or similar. As usual in DBR, a mixed-method approach of data collection was chosen. Quantitative data was 
collected using questionnaires on cognitive load and acceptance of AR technology; qualitative data was collected through group discussions.

To measure cognitive load, we used the Nasa Task Load Index (Nasa-TLX) (Hart, 2006), the questionnaire used most frequently to measure cognitive load in human-computer studies, because it allows the multidimensional assessment of cognitive load (Grier, 2015). The subscales survey participants' perceptions regarding mental, physical and temporal demands, performance, effort and frustration. Together they add up to the overall cognitive load.

To find out whether the AR learning environment we had designed was acceptable to the participants, we used an adapted version of the Technology Usage Inventory (TUI) developed by Kothgassner et al. (2013). This version includes the scales for the Usability and Usefulness of AR for learning, and for Skepticism towards the use of AR in learning environments. In addition, we also asked participants whether they would be happy to continue using AR in other learning settings. They completed the questions on a scale of 1 (strongly disagree) to 7 (strongly agree). An example of an item from the usefulness scale is 'The use of augmented reality could help me to complete the learning tasks more efficiently'.

\subsection{Procedure}

The trial session took place in summer 2020 and complied with the COVID-related hygiene requirements. At the beginning of the trial, the participants were welcomed and briefly introduced to the AR application. Subsequently, the four AR modules were tested collaboratively. At the end, students completed the questionnaire, which included TUI as well as Nasa-TLX items.

\subsection{Data analysis}

The data was processed and analysed using the statistical software SPSS 27. Scales were first calculated using items from the TUI sub-scales (e.g., the usability scale was formed from three items on the use of the AR application). In total, the four scales Usability, Usefulness, Skepticism and Intention to Use were evaluated; Cronbach's alpha ranged from 0.6 to 0.8, which indicates sufficient reliability of the measurement instrument. The six items from the Nasa-TLX were aggregated into an overall scale of cognitive load, for which Cronbach's alpha $=0.8$. The Performance sub-scale was recoded according to the questionnaire design; low values here stand for a positive assessment of one's own performance during learning in the AR environment.

\section{$5 \quad$ Results}

\subsection{Cognitive load}

Table 2 shows the mean values with standard deviation for the sub-scales from the Nasa-TLX and the total cognitive load scale. The participants perceived the AR learning environment as not cognitively demanding; the overall cognitive load was reported as being low, with $\mathrm{M}=$ $3.17(\mathrm{SD}=1.51)$. This is also reflected in the values of the sub-scales; for example, the learners 


\section{Buchner et al}

did not feel any time pressure or excessive physical strain. The values for the Performance scale $(\mathrm{M}=3.08, \mathrm{SD}=1.38)$ indicate that the learners were able to learn successfully with the help of the AR modules.

Table 1: Descriptive statistics of the Nasa-TLX scales with means and standard deviations.

\begin{tabular}{lll}
\hline Variable & M SD \\
\hline Mental Demand & 4.42 & 2.07 \\
Physical Demand & 2.50 & 2.20 \\
Temporal Demand & 2.92 & 2.61 \\
Performance & 3.08 & 1.38 \\
Effort & 2.75 & 2.34 \\
Frustration & 3.33 & 2.67 \\
& & \\
Overall Cognitive Load & 3.17 & 1.51 \\
\hline Note: Scales range from 1 to 10. Scale Performance is reverse-coded.
\end{tabular}

\subsection{Acceptance of the technology}

In terms of its technical requirements, the AR learning environment was predominantly rated positively by participants, with Usability $(\mathrm{M}=6.08, \mathrm{SD}=0.64)$ being rated a little higher than Usefulness $(\mathrm{M}=5.56, \mathrm{SD}=0.70)$ (see Table 3). The value for the Skepticism scale $(\mathrm{M}=3.50$, $\mathrm{SD}=0.69)$ is in the middle of the seven-point Likert-scale, so there were different opinions within the group. The results for Intention to Use $(\mathrm{M}=5.42, \mathrm{SD}=1.02)$ allow the conclusion that the participants' willingness to use AR in the context of future learning activities is high.

Table 3: Descriptive statistics of the TUI scales, with means and standard deviations.

\begin{tabular}{lll}
\hline Variable & M & SD \\
\hline Usability & 6.08 & 0.64 \\
Usefulness & 5.56 & 0.70 \\
Skepticism & 3.50 & 0.69 \\
& & \\
Intention to Use & 5.42 & 1.02 \\
\hline
\end{tabular}

Note: Scales range from 1 to 7 . 


\section{Discussion and outlook}

In this study, we have presented the development and design of four AR modules in the context of an informal educational programme in geoinformatics. We considered the requirement for active learning in science education as well as the potential risks of using AR in education and based our pedagogical framework on this. In order not to overburden students in an AR learning environment, we focused on collaborative learning. The individual elements in the AR modules were designed in such a way that they do not overwhelm learners; rather, they enable students to learn in a holistic, interactive way (behaviourally, cognitively and emotionally). The results of the evaluation regarding cognitive load reflect the careful planning that went into our design: the participants did not experience cognitive overload in this first pilot. They rated their own performance on the tasks to be completed using AR as high. These results are in line with other studies on the use of AR. For example, Turan et al. (2018) showed that an evidence-informed AR learning environment in geography was not perceived as cognitively demanding. Lin et al. (2015) draw the same conclusion for AR learning in geometry, especially when learning is collaborative.

For the technical implementation, we used marker-based AR technology. The combination of Wikitude and Unity proved to be successful. We were able to create the three-dimensional and animated visualizations necessary for understanding the content of the modules, and to make them accessible via mobile devices without technical difficulties. The evaluation showed that this form of presentation was perceived as useful and user-friendly. The willingness to continue learning using AR was very high, and scepticism about AR as an educational medium was low.

Although our results demonstrate that the use of AR in informal learning settings is very much welcomed, we should also point out that such learning opportunities would have to be made accessible for all schools - not only for privileged schools but for everyone, avoiding any further technological and social divides. Consideration of this social aspect was essential for us and our ARED project. First, we learnt that informal learning spaces can be of particular importance in the educational process. It is all the more essential that educational institutions present innovative concepts and make these available in schools. This is also the conclusion of Yoon et al. (2017), who used AR installations in various museums. Secondly, we took away from our experience that a project like this should also think about how we can access schools directly. Mund and Müller (2019) report how difficult it can be to integrate AR-supported learning offers into the regular classroom. We are taking this as a mission and intend to create AR-enhanced learning materials that can be used regardless of time and place. We will also further revise the AR modules. For example, it became clear that the construction task in one module (see Appendix: Citizen sensing platform) could be improved if the learners had their hands completely free for the task. Here, the use of AR glasses would be a possible improvement, which we would like to test in the next iteration. In addition, we will include a control group in the next run to test our pedagogical framework. For example, we will compare individual vs. collaborative learning using the AR modules. In our next study, we also plan to record other variables, e.g. learning achievement, task performance or motivation. In this study, only cognitive load and acceptance were investigated, which must be considered a limitation. A further limitation of this first prototype study is the small sample size. The results must therefore be interpreted with caution; further studies are required to learn more about 
the effects of our design. Additionally, in a future study we will test the learning environment with school students in order to gain insights into how to improve the design for this target group.

In summary, the possible uses of $A R$ in geoinformatics education go beyond those of mere visualization of complex phenomena. Our project shows that with the help of an AR learning environment, designed by a multidisciplinary team, holistic learning experiences can be initiated that learners are happy to engage in and do not perceive as overwhelming. Informal educational institutions can use AR-based pedagogical approaches as a welcome alternative to everyday school life to inspire students for science and research.

\section{Acknowledgement}

We would like to thank the volunteers who made the evaluation of the first implementation possible. We would especially like to thank Philipp Nagele from Wikitude, who allowed us to use Wikitude AR and supported us in creating the AR objects.

\section{Appendix}

Module: Performing geospatial tasks with the augmented reality sandbox

The AR Sandbox has a built-in AR component, which is the main element of this learning environment. The equipment consists of a computer and a projector, as well as a structured light range sensor mounted above a sand surface (in our case, a Microsoft Kinect for XBOX 360). In a structured light-based approach, the Kinect emits a narrow band of light towards a surface. The surface relief, in this case a sand landscape, changes the patterns of light and shade, which are observed from multiple cameras at different positions on the Kinect device. The distortion between the expected and the observed pattern is used to calculate the physical offsets on the surface, in this case the depth or height of the surface relief. Using the calculated height, an isopleth map is rendered in the backend computer and then projected onto the sand surface, thus visualizing a digital elevation model. Additional interaction capabilities are implemented based on object recognition.

The sandbox offers a broad range of possibilities to perform small-scale landscape modelling in an interactive way, based on subjective decisions and actions. Using this technology without additional instructions, however, limits the capabilities and learning targets. For example, no classification can be derived from the colour assignment of the corresponding heights. Consequently, it is difficult to assess the heights of the summits in the sand landscape in relation to each other, which is an obstacle in landscape modelling. Thus, the goal of our study is to provide additional information and learning tasks in an AR-based application in order to improve the communication of height information. This additional information is displayed in written form and superimposed on the surface (see Figure 3, right). It allows better understanding of height differences and thus also allows more realistic landscape modelling through the representation of elevation scales. 
A critical question in the implementation of this use-case was information density. The existing AR component of the sandbox requires some further information in order not to draw attention away from the application itself. For this reason, we decided to illustrate aggregated elevation levels using multiple markers. Depending on the elevation levels, markers were designed as triggers for displaying specific, locally relevant information. For example, for elevations between 706 and 1,519 metres, the Gaisberg mountain was set as the reference. In total, we created descriptions for eight markers, representing the height of different topographical features like mountains or lakes. After the AR app has recognized the marker, the information is displayed without any additional user interaction. Figure 3 shows the markers for the altitude levels (left), and the exemplary application (right). A video demonstration of the sandbox module is available here: t1p.de/sandbox1
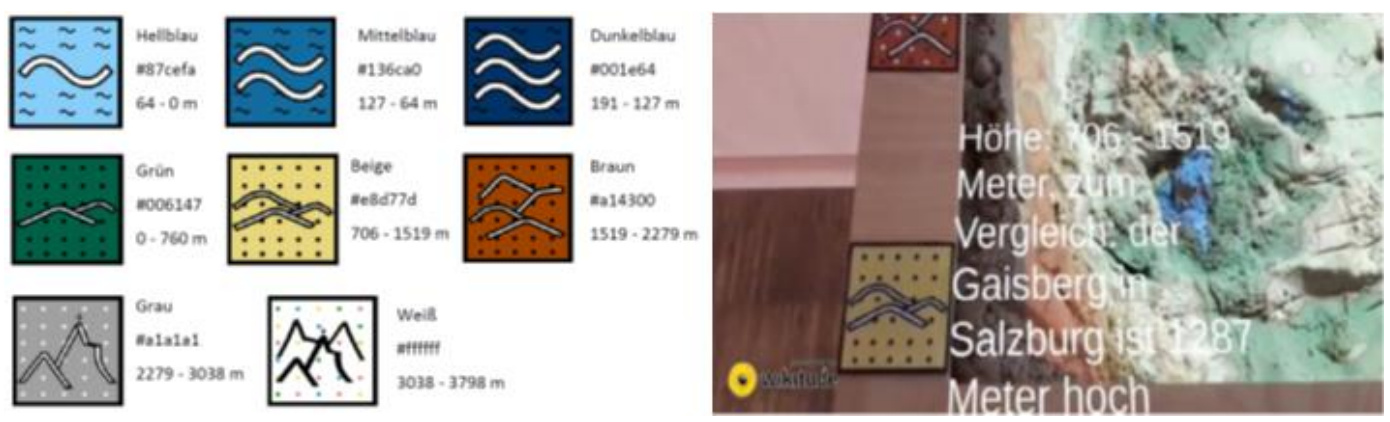

Figure 3: Markers (left) and demonstration of the additional information superimposed on the sandbox (right).

\section{Module: AR-based construction guidance for a Citizen Sensing platform}

In this module, learners are introduced to measurable environmental phenomena on the one hand, and to the concepts of Citizen Sensing more broadly on the other. Learners use our AR app to assemble a sensing station in a self-guided manner. For this, we used the senseBox:Edu, which was designed to enable the construction and operation of different sensing stations with a variety of measurement parameters and a broadly variable degree of difficulty. The hardware kit used consists of (often combined) sensors for different measurement variables, various connection cables, a circuit board and the central Micro Control Unit (MCU). For advanced learners, the measurement application can be programmed in the MCU itself. The measurement setup includes pre-designed instructions for specific measurement stations. This means alternating attention between the instructions that need to be read and understood, and the measurement hardware that is being assembled. This continuously shifting attention is a typical source of errors and increases the time needed to complete the task of assembling the sensing station. Furthermore, the various sensors require close inspection for identification because of similarities in appearance. Therefore, the goal of this scenario is to keep the learner's focus on the senseBox (Figure 4, left) with the help of the presentation of AR-based information. Installation steps are interactively displayed in the AR environment through object identification; individual sensors are identified by their shape and texture. To reach these goals, we designed a dual approach to augmentation. First, the installation instructions (several 
consecutive steps) were transferred to virtual text objects. For this marker-based approach, the step numbers of the installation were used as triggers. As soon as the AR app recognizes a marker, it initializes the display of the current installation step. These basic-level steps include the simple identification of hardware pieces, for which the second approach was designed: previously generated digital models of each element of the hardware were used to enable the recognition-based identification of objects. This means that the actual object (a sensor, the MCU, the display, etc.) were recognized by the AR app. The combination of these two approaches leads to an AR-based installation tutorial that includes hardware identification, preventing the learner from making assembly errors and hardware mix-ups. The two approaches with sensor identification (centre) and AR-based manual (right) are shown in Figure 4. A video demonstration of the senseBox module is available here: t1p.de/sensebox

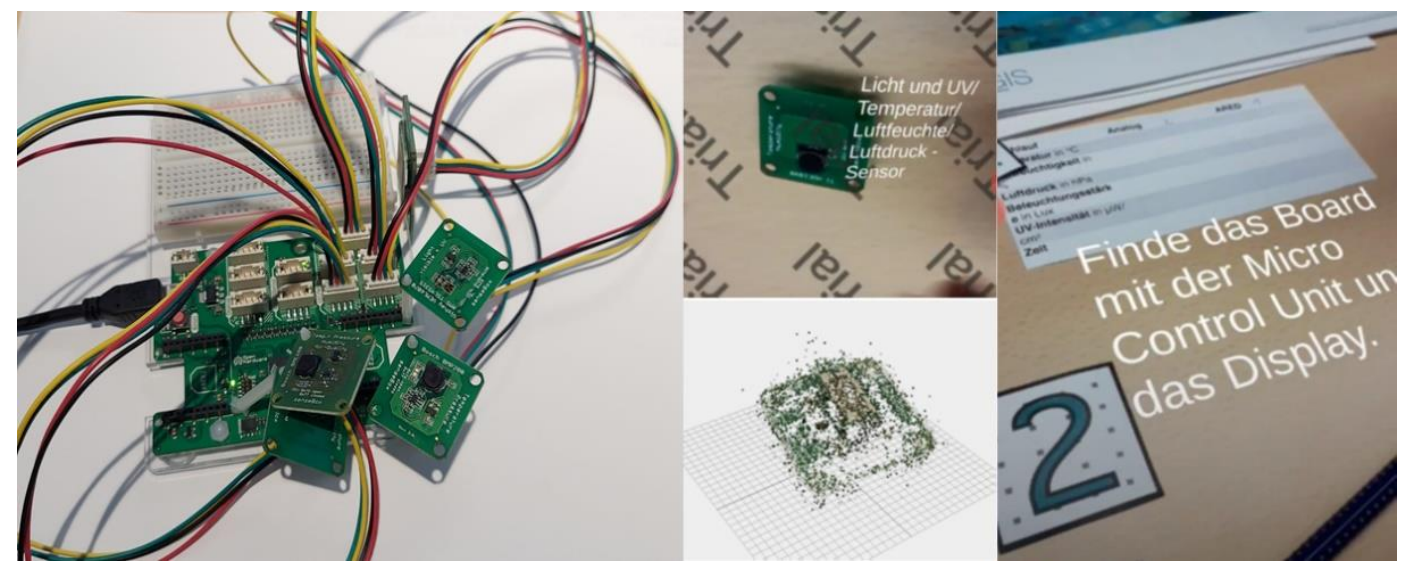

Figure 4: SenseBox (left), with sensor identification (centre), and the AR-based construction manual (right).

\section{References}

Ayres, P., \& Sweller, J. (2014). The Split-Attention Principle in Multimedia Learning. In R. E. Mayer (Ed.), The Cambridge Handbook of Multimedia Learning (Second Edition, pp. 206-226). Cambridge University Press.

Azuma, R., Baillot, Y., Behringer, R., Feiner, S., Julier, S., \& MacIntyre, B. (2001). Recent advances in augmented reality. IEEE Computer Graphics and Applications, 21(6), 34-47. https://doi.org/10.1109/38.963459

Bell, P. (2004). On the Theoretical Breadth of Design-Based Research in Education. Educational Psychologist, 39(4), 243-253. https://doi.org/10.1207/s15326985ep3904_6

Chang, S.-C., \& Hwang, G.-J. (2018). Impacts of an augmented reality-based flipped learning guiding approach on students' scientific project performance and perceptions. Computers \& Education, 125, 226-239. https://doi.org/10.1016/j.compedu.2018.06.007

Cheng, K.-H., \& Tsai, C.-C. (2013). Affordances of Augmented Reality in Science Learning: Suggestions for Future Research. Journal of Science Education and Technology, 22(4), 449-462. https://doi.org/10.1007/s10956-012-9405-9 
Deslauriers, L., Schelew, E., \& Wieman, C. (2011). Improved Learning in a Large-Enrollment Physics Class. Science, 332(6031), 862-864. https://doi.org/10.1126/science.1202043

Domagk, S., Schwartz, R. N., \& Plass, J. L. (2010). Interactivity in multimedia learning: An integrated model. Computers in Human Behavior, 26(5), 1024-1033. https://doi.org/10.1016/j.chb.2010.03.003

Dunleavy, M., Dede, C., \& Mitchell, R. (2009). Affordances and Limitations of Immersive Participatory Augmented Reality Simulations for Teaching and Learning. Journal of Science Education and Technology, 18(1), 7-22. https://doi.org/10.1007/s10956-008-9119-1

Goff, E. E., Hartstone-Rose, A., Irvin, M. J., \& Mulvey, K. L. (2020). Using Augmented Reality to Promote Active Learning in College Science. In J. J. Mintzes \& E. M. Walter (Eds.), Active Learning in College Science (pp. 741-755). Springer International Publishing. https://doi.org/10.1007/978-3-030-33600-4_46

Grier, R. A. (2015). How High is High? A Meta-Analysis of NASA-TLX Global Workload Scores. Proceedings of the Human Factors and Ergonomics Society Annual Meeting, 59(1), 1727-1731. https://doi.org/10.1177/1541931215591373

Hart, S. G. (2006). NASA-Task Load Index (NASA-TLX); 20 Years Later. Proceedings of the Human Factors and Ergonomics Society 50th Annual Meeting, 904-908.

Ibáñez, M.-B., \& Delgado-Kloos, C. (2018). Augmented reality for STEM learning: A systematic review. Computers \& Education, 123, 109-123. https://doi.org/10.1016/j.compedu.2018.05.002

Ibili, E., Resnyansky, D., \& Billinghurst, M. (2019). Applying the technology acceptance model to understand maths teachers' perceptions towards an augmented reality tutoring system. Education and Information Technologies, 24(5), 2653-2675. https://doi.org/10.1007/s10639-019-09925-z

Janssen, J., \& Kirschner, P. A. (2020). Applying collaborative cognitive load theory to computersupported collaborative learning: Towards a research agenda. Educational Technology Research and Development, 68(2), 783-805. https://doi.org/10.1007/s11423-019-09729-5

Kothgassner, O. D., Felnhofer, A., Hauk, N., Kastenhofer, E., Gomm, J., \& Kryspin-Exner, I. (2013). TUI: Technology Usage Inventory. https://www.ffg.at/sites/default/files/allgemeine_downloads/thematische\%20programme/progr ammdokumente/tui_manual.pdf

Lai, A.-F., Chen, C.-H., \& Lee, G.-Y. (2019). An augmented reality-based learning approach to enhancing students' science reading performances from the perspective of the cognitive load theory. British Journal of Educational Technology, 50(1), 232-247. https://doi.org/10.1111/bjet.12716

Lin, H.-C. K., Chen, M.-C., \& Chang, C.-K. (2015). Assessing the effectiveness of learning solid geometry by using an augmented reality-assisted learning system. Interactive Learning Environments, 23(6), 799-810. https://doi.org/10.1080/10494820.2013.817435

Lindgren, R., Tscholl, M., Wang, S., \& Johnson, E. (2016). Enhancing learning and engagement through embodied interaction within a mixed reality simulation. Computers \& Education, 95, 174187. https://doi.org/10.1016/j.compedu.2016.01.001

McKenney, S., \& Reeves, T. C. (2019). Conducting Educational Design Research (2.). Roudledge.

Mund, J.-P., \& Müller, S. (2019). Augmented Reality and Mobile GIS as Tools for Teaching Datacollection in the Context of Forest Inventories. GI_Forum, 1, 129-143. https://doi.org/10.1553/giscience2019_02_s129

Resch, B., Summa, A., Zeile, P., \& Strube, M. (2016). Citizen-Centric Urban Planning through Extracting Emotion Information from Twitter in an Interdisciplinary Space-Time-Linguistics Algorithm. Urban Planning, 1(2), 114-127. https://doi.org/10.17645/up.v1i2.617

Sahin, D., \& Yilmaz, R. M. (2020). The effect of Augmented Reality Technology on middle school students' achievements and attitudes towards science education. Computers \& Education, 144, 103710. https://doi.org/10.1016/j.compedu.2019.103710 
Sommerauer, P., \& Müller, O. (2014). Augmented reality in informal learning environments: A field experiment in a mathematics exhibition. Computers \& Education, 79, 59-68. https://doi.org/10.1016/j.compedu.2014.07.013

Sweller, J., van Merriënboer, J., \& Paas, F. G. W. C. (2019). Cognitive Architecture and Instructional Design: 20 Years Later. Educational Psychology Review, 31(2), 261-292. https://doi.org/10.1007/s10648-019-09465-5

Teunissen, P. J. G., \& Montenbruck, O. (Eds.). (2017). Springer Handbook of Global Navigation Satellite Systems. Springer International Publishing. https://doi.org/10.1007/978-3-319-42928-1

Turan, Z., Meral, E., \& Sahin, I. F. (2018). The impact of mobile augmented reality in geography education: Achievements, cognitive loads and views of university students. Journal of Geography in Higher Education, 42(3), 427-441. https://doi.org/10.1080/03098265.2018.1455174

Wikitude. (2021). Wikitude Augmented Reality SDK. Wikitude. https://www.wikitude.com/

Yannier, N., Hudson, S. E., \& Koedinger, K. R. (2020). Active Learning is About More Than HandsOn: A Mixed-Reality AI System to Support STEM Education. International Journal of Artificial Intelligence in Education, 30(1), 74-96. https://doi.org/10.1007/s40593-020-00194-3

Yoon, S., Anderson, E., Lin, J., \& Elinich, K. (2017). How Augmented Reality Enables Conceptual Understanding of Challenging Science Content. Educational Technology \& Society, 20(1), 156168. 